ВІСНИК

ОДЕСЬКОГО НАЦІОНАЛЬНОГО

МОРСЬКОГО УНІВЕРСИТЕТУ

№ 2 (65), 2021
HERALD

OF THE ODESSA NATIONAL

MARITIME UNIVERSITY № 2 (65), 2021

УДК 620:178.3

DOI 10.47049/2226-1893-2021-2-120-127

\title{
ОЦЕНКА ВЛИЯНИЯ СКОРОСТИ УВЕЛИЧЕНИЯ НАГРУЗКИ НА ТОЧНОСТЬ ОПРЕДЕЛЕНИЯ РАЗРУШАЮЩЕГО НАПРЯЖЕНИЯ С ПОМОЩЬЮ ГИПОТЕЗЫ ЛИНЕЙНОГО СУММИРОВАНИЯ УСТАЛОСТНЫХ ПОВРЕЖДЕНИЙ
}

А.В. Коноплёв

д.т.н., профессор, заведующий кафедрой «Машиноведение»

А.Н. Шумило

к.т.н., професор, доцент кафедры «Машиноведение»

Н.И. Чередарчук

старший преподаватель кафедры «Физика, математика и астрономия»

В.В. Галевский, О.Н. Кононова

старшие преподаватели кафедры «Машиноведение» Е.К. Рожко

ассистент кафедры «Прикладная механика»

В.А. Арпентьева

аспирант кафедры «Машиноведение»

Одесский национальный морской университет, Одесса, Украина

Аннотация. С помощью линейной гипотезы накопления усталостных повреждений рассчитаны значения разрушаюшчих напряжений при имитацчи испьтаний деталей машин на сопротивление усталости с линейно увеличивающейся нагрузкой. Расчёты проведены для диапазона дискретных значений предела выносливости в диапазоне 100500 МПа и скоростей увеличения амплитуды напряжений 100-500 Па/цзикл.

Расчёт показал, что погрешность разрушающих напряжений зависит от скорости увеличения нагрузки и в предельном случае не превынает 15 \%.

Ключевые слова: накопление усталостных повреждений, скорость увеличения нагрузки, разрушающее напряжение.

УДК 620:178.3

DOI 10.47049/2226-1893-2021-2-120-127

\section{ОЦІНКА ВПЛИВУ ШВИДКОСТІ ЗБІЛЬШЕННЯ НАВАНТАЖЕННЯ \\ НА ТОЧНІСТЬ ВИЗНАЧЕННЯ РУЙНІВНОГО НАПРУЖЕННЯ \\ ЗА ДОПОМОГОЮ ГІПОТЕЗИ ЛІНІЙНОГО ПІДСУМОВУВАННЯ ВТОМНИХ ПОШКОДЖЕНЬ}

А.В. Конопльов

д.т.н., професор, завідувач кафедри «Машинознавство»

О.М. Шумило

к.т.н, професор, доцент кафедри «Машинознавство»

$$
\text { Н.I. Чередарчук }
$$

старший викладач кафедри «Фізика, математика та астрономія»

В.В. Галевський, О.М. Кононова

старші викладачі кафедри «Машинознавство»

О.К. Рожко

асистент кафедри «Прикладна механіка»

В.О. Арпентьєва

аспірант кафедри «Машинознавство»

Одеський національний морський університет, Україна, Одеса

() Коноплев А.В., Шумило А.Н., Чередарчук Н.И., Галевский В.В., Кононова О.Н., Рожко Е.К., Арпентьева В.А., 2021 
Анотація. За допомогою лінійної гіпотези накопичення втомних пошкоджень розраховані значення руйнівних напружень при імітації випробувань деталей машин на опір втоми з навантаженням щзо збільиується лінійно. Розрахунки проведені для діапазону дискретних значень межі витривалості в діапазоні 100-500 МПа і швидкостей збільшення амплітуди напруг 100-500 Па/циикл.

Розрахунок показав, щчо похибка руйнівних напружень залежить від швидкості збільшення навантаження і в граничному випадку не перевимуе $15 \%$.

Ключові слова: накопичення втомних пошкоджень, швидкість збільшення навантаження, руйнівне напруження.

UDC 620:178.3

DOI 10.47049/2226-1893-2021-2-120-127

\title{
EVALUATION OF THE EFFECT OF THE RATE OF INCREASE IN THE LOAD ON THE ACCURACY OF DETERMINING THE BREAKING TENSIONS USING THE HYPOTHESIS OF LINEAR SUMMATION OF FATIGUE DAMAGES
}

\author{
A.V. Konoplev \\ Doctor of Technical Sciences, Professor, \\ Head of the Department of «Machine Science» \\ A.N. Shumilo
}

Ph.D. Associate Professor of the Department of «Machine Science»

N.I. Cheredarchuk

Senior Lecturer of the Department «Mathematics, physics and astronomy»

V.V. Halevskyi, O.N. Kononova

Senior Lecturers of the Department «Machine Science»

\section{E.K. Rozhko}

Assistant of the Department «Applied mechanics»

V.O. Arpentieva

Postgraduate student of the department «Machine Science»

Odessa National Maritime University, Odessa, Ukraine

Abstract. Using the linear hypothesis of fatigue damage accumulation, the values of breaking tensions are calculated when simulating fatigue resistance tests of machine parts with a linearly increasing load. The calculations were carried out for the range of discrete values of the endurance limit in the range of 100-500 $\mathrm{MPa}$ and the rates of increase in the stress range 100-500 Pa/cycle.

The calculation showed that the error of the breaking tensions depends on the rate of increase in the load and, in the limiting case, does not exceed $15 \%$.

Keywords: accumulation of fatigue damage, load increase rate, breaking tension. 
Введение. Ускоренные испытания на сопротивление усталости чаще всего проводят при возрастающей амплитуде напряжений, что предполагает использование той или иной теории накопления усталостных повреждений. В настоящее время существует несколько таких теорий, каждая из которых имеет свои преимущества и недостатки [1]. Наиболее простой и самой распространённой считают линейную гипотезу суммирования усталостных повреждений, которая была предложена Пальмгреном и Майнером ещё в 1924 году.

Согласно данной гипотезе воздействие нестационарной нагрузки приведёт к повреждённостям $D_{i}$, а разрушение произойдет, когда сумма этих долей повреждённости составит единицу [1]

$$
D_{1}+D_{2}+\ldots+D_{i-1}+D_{i}=1 \text {. }
$$

Доля повреждённости, согласно линейной гипотезе, прямо пропорциональна отношению числа циклов $n_{i}$ к полному числу циклов $N_{i}$, которое привело бы к разрушению, т.е.

$$
D_{i}=\frac{n_{i}}{N_{i}}
$$

Многочисленные проверки формулы (1) показали, что сумма долей повреждённости хотя и близка к единице, но зависит от ряда факторов и в первую очередь от режима нагружения (чередования убывания или возрастания нагрузки). Учитывая данный факт, а также зависимость (1) формулу (2) можно записать в общем виде

$$
\frac{n_{1}}{N_{1}}+\frac{n_{2}}{N_{2}}+\ldots+\frac{n_{i-1}}{N_{i-1}}+\frac{n_{i}}{N_{i}}=\sum_{i=1}^{i} \frac{n_{j}}{N_{j}}=a
$$

Рассматривая частный случай нагружения объектов, а именно, случай испытаний при линейно возрастающей нагрузке $\alpha$, выражение (3) будет иметь вид

$$
\int_{0}^{n_{c}} d n / N=\left(\int_{\sigma_{1}}^{\sigma_{P}} d \sigma / N\right) / \alpha=a .
$$


Опытным путём установлено, что значение величины $a$ приближается к единице при квазислучайном нагружении, но в случае, когда нагрузка только возрастает или убывает, её значение может колебаться в пределах 0,6-1,6 [1]. При этом изменение её величины в ту тли иную сторону от единицы не зависит от режима нагружения и не носит закономерный характер.

Целью статьи является оценка влияния суммы накопленных повреждений $a$ на погрешность определения разрушающего напряжения для диапазона скоростей увеличения нагрузки $100-500$ Па/цикл и диапазона дискретных значений предела выносливости 100-500 МПа.

Изложение основного материала. Для проведения исследования примем в качестве модели кривой усталости уравнение Вейбулла, которое запишем в виде

$$
\left(\sigma-\sigma_{R}\right)^{m_{W}} \cdot N=10^{C_{W}}
$$

где $\sigma$ и $N$ - текущие значения напряжений и долговечности до разрушения соответственно;

$\sigma_{R}-$ предел выносливости;

$m_{W}$ и $C_{W}-$ параметры.

Зависимость для определения разрушающего напряжения при линейном увеличении нагрузки от начального напряжения, не превышающего предел выносливости, получим путём совместного решения уравнений (4) и (5). В результате имеем

$$
\sigma_{P}=\sigma_{R}+\left[a \alpha(m+1) \cdot 10^{C_{W}}\right]^{\frac{1}{m_{W}+1}}
$$

Для проведения расчёта зададимся дискретными значениями предела выносливости в диапазоне 100-500 МПа (через каждые 100 МПа). При этом параметры $m_{W}$ и $C_{W}$, входящие в уравнение (9), будем определять с помощью корреляционных уравнений, связывающих их с пределом выносливости [2]

$$
\begin{gathered}
m_{W}=\frac{1}{5,254 \sigma_{R}^{0,01}-5,038}-1 ; \\
\tilde{N}_{W}=\lg \frac{\left(7,029 \sigma_{R}+3599\right)^{m_{W}+1}}{m_{W}+1} .
\end{gathered}
$$


ВІСНИК

ОДЕСЬКОГО НАЦІОНАЛЬНОГО МОРСЬКОГО УНІВЕРСИТЕТУ № 2 (65), 2021
HERALD

OF THE ODESSA NATIONAL

MARITIME UNIVERSITY

Результаты расчёта разрушающих напряжений $\sigma_{P}$ и их погрешностей $\delta_{a}$ при значениях $a$ равных 0,$6 ; 0,8 ; 1,0 ; 1,2 ; 1,4$ и 1,6 представлены в таблице 1.

Таблица 1

Расчётные значения разрушающих напряжений $\sigma_{P} и$ их погрешностей $\delta_{\mathrm{a}}$ в зависимости от суммы накопленных повреждений $a$

\begin{tabular}{|c|c|c|c|c|c|c|c|c|c|c|c|}
\hline \multirow{2}{*}{$\begin{array}{c}\sigma_{R}, \\
\mathrm{M \Pi а}\end{array}$} & \multicolumn{6}{|c|}{$\sigma_{P}, M П а$ при $а$ равном } & \multicolumn{5}{|c|}{$\delta_{\mathrm{a}}, \%$ при $a$ равном } \\
\hline & 0,6 & 0,8 & 1,0 & 1,2 & 1,4 & 1,6 & 0,6 & 0,8 & 1,2 & 1,4 & 1,6 \\
\hline & \multicolumn{11}{|c|}{$\alpha=100$ Па/цикл } \\
\hline 100 & 147,5 & 154,2 & 160,1 & 165,5 & 170,3 & 174,8 & $-7,9$ & $-3,7$ & 3,3 & 6,3 & 9,1 \\
\hline 200 & 238,1 & 244,0 & 249,2 & 253,9 & 258,2 & 262,3 & $-4,5$ & $-2,1$ & 1,9 & 3,6 & 5,3 \\
\hline 300 & 334,9 & 340,6 & 345,6 & 350,2 & 354,4 & 358,3 & $-3,1$ & $-1,5$ & 1,3 & 2,5 & 3,7 \\
\hline 400 & 433,5 & 439,2 & 444,2 & 448,8 & 453,0 & 457,0 & $-2,4$ & $-1,1$ & 1,0 & 2,0 & 2,9 \\
\hline \multirow[t]{2}{*}{500} & 533,0 & 538,6 & 543,7 & 548,3 & 552,6 & 556,7 & $-2,0$ & $-0,9$ & 0,9 & 1,6 & 2,4 \\
\hline & \multicolumn{11}{|c|}{$=200$ Па/цикл } \\
\hline 100 & 165,5 & 174,8 & 182,9 & 190,3 & 196,9 & 203,1 & $-9,6$ & $-4,5$ & 4,0 & 7,7 & 11,0 \\
\hline 200 & 253,9 & 262,3 & 269,7 & 276,3 & 282,5 & 288,2 & $-5,8$ & $-2,7$ & 2,5 & 4,8 & 6,9 \\
\hline 300 & 350,2 & 358,3 & 365,6 & 372,2 & 378,2 & 383,9 & $-4,2$ & $-2,0$ & 1,8 & 3,5 & 5,0 \\
\hline 400 & 448,8 & 457,0 & 464,3 & 470,9 & 477,1 & 482,8 & $-3,3$ & $-1,6$ & 1,4 & 2,8 & 4,0 \\
\hline \multirow[t]{2}{*}{500} & 548,3 & 556,7 & 564,1 & 570,9 & 577,2 & 583,2 & $-2,8$ & $-1,3$ & 1,2 & 2,3 & 3,4 \\
\hline & \multicolumn{11}{|c|}{$=300$ Па/цикл } \\
\hline 100 & 179,0 & 190,3 & 200,1 & 208,9 & 217,0 & 224,5 & $-10,5$ & $-4,9$ & 4,4 & 8,4 & 12,2 \\
\hline 200 & 266,1 & 276,3 & 285,4 & 293,6 & 301,1 & 308,1 & $-6,8$ & $-3,2$ & 2,9 & 5,5 & 8,0 \\
\hline 300 & 362,1 & 372,2 & 381,1 & 389,3 & 396,8 & 403,8 & $-5,0$ & $-2,4$ & 2,1 & 4,1 & 5,9 \\
\hline 400 & 460,7 & 470,9 & 480,0 & 488,3 & 496,0 & 503,1 & $-4,0$ & $-1,9$ & 1,7 & 3,3 & 4,8 \\
\hline \multirow[t]{2}{*}{500} & 560,5 & 570,9 & 580,2 & 588,7 & 596,6 & 604,0 & $-3,4$ & $-1,6$ & 1,5 & 2,8 & 4,1 \\
\hline & \multicolumn{11}{|c|}{$=400$ Па/цикл } \\
\hline 100 & 190,3 & 203,1 & 214,4 & 224,5 & 233,7 & 242,2 & $-11,3$ & $-5,2$ & 4,7 & 9,0 & 13,0 \\
\hline 200 & 276,3 & 288,2 & 298,6 & 308,1 & 316,8 & 324,9 & $-7,5$ & $-3,5$ & 3,2 & 6,1 & 8,8 \\
\hline 300 & 372,2 & 383,9 & 394,3 & 403,8 & 412,5 & 420,7 & $-5,6$ & $-2,6$ & 2,4 & 4,6 & 6,7 \\
\hline 400 & 470,9 & 482,8 & 493,5 & 503,1 & 512,1 & 520,5 & $-4,6$ & $-2,2$ & 2,0 & 3,8 & 5,5 \\
\hline \multirow[t]{2}{*}{500} & 570,9 & 583,2 & 594,1 & 604,0 & 613,3 & 622,0 & $-3,9$ & $-1,8$ & 1,7 & 3,2 & 4,7 \\
\hline & \multicolumn{11}{|c|}{$\alpha=500$ Па/цикл } \\
\hline 100 & 200,1 & 214,4 & 226,8 & 238,0 & 248,3 & 257,7 & $-11,8$ & $-5,5$ & 4,9 & 9,4 & 13,6 \\
\hline 200 & 285,4 & 298,6 & 310,3 & 320,9 & 330,6 & 339,7 & $-8,0$ & $-3,8$ & 3,4 & 6,5 & 9,5 \\
\hline 300 & 381,1 & 394,3 & 406,0 & 416,7 & 426,5 & 435,7 & $-6,1$ & $-2,9$ & 2,6 & 5,0 & 7,3 \\
\hline 400 & 480,0 & 493,5 & 505,4 & 516,4 & 526,5 & 535,9 & $-5,0$ & $-2,4$ & 2,2 & 4,2 & 6,0 \\
\hline 500 & 580,2 & 594,1 & 606,4 & 617,7 & 628,2 & 638,0 & $-4,3$ & $-2,0$ & 1,9 & 3,6 & 5,2 \\
\hline
\end{tabular}


Отклонения разрушающих напряжений $\delta_{a}$ в зависимости от суммы накопленных повреждений $a$ находили по формуле

$$
\delta_{a}=\frac{\sigma_{P a}-\sigma_{P 1,0}}{\sigma_{P 1,0}} \cdot 100,
$$

где $\sigma_{P a}$ - разрушающее напряжение, соответствующее определённому значению суммы накопленных повреждений $a$;

$\sigma_{P 1,0}$ - разрушающее напряжение, соответствующее значению $a=1$.

Рассчитанные по формуле (9) значения отклонений $\delta_{a}$ в зависимости от суммы накопленных повреждений $a$ для скоростей $\alpha=100$ и 500 Па/цикл изображены на рис. 1 и 2. При скоростях $\alpha$, равных 200, 300 и 400 Па/цикл величины $\delta_{a}$ занимают промежуточные значения, поэтому их графики не приводятся.

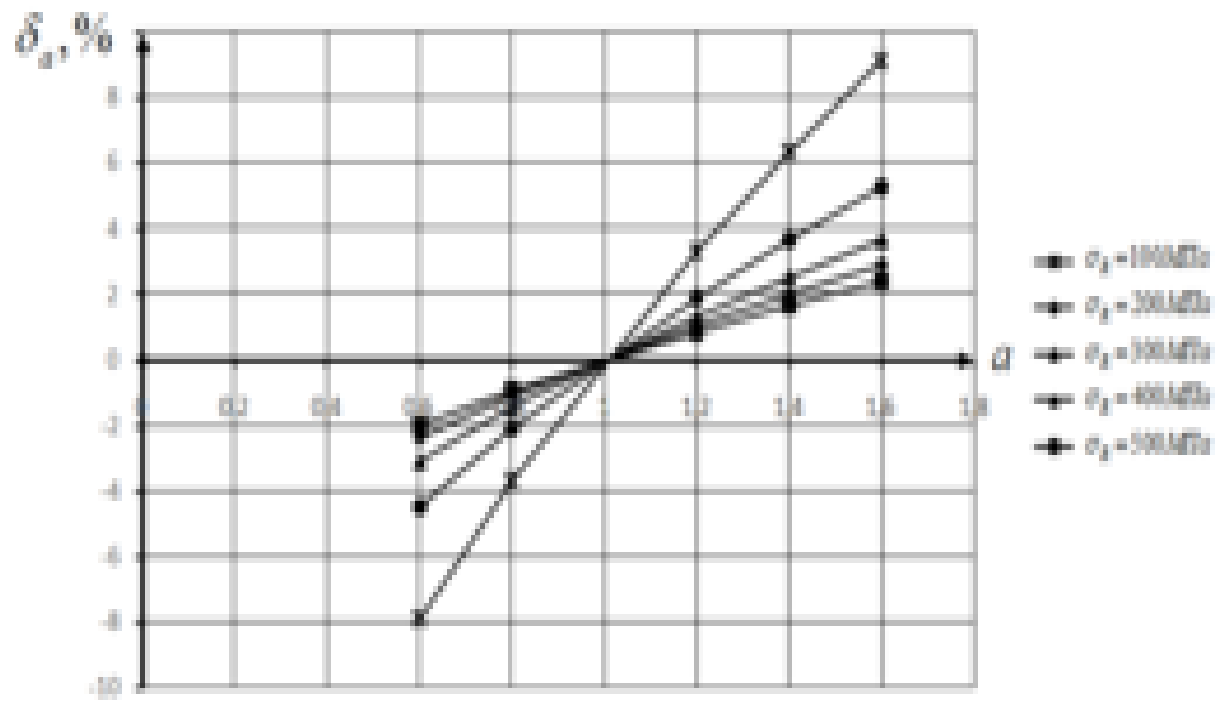

Рис. 1. Зависимости отклонения разрушающего напряжения $\delta_{a}$ от суммы накопленных повреждений а при скорости роста напряжений $\alpha=100$ Па/иякл 
ВІСНИК

ОДЕСЬКОГО НАЦІОНАЛЬНОГО

МОРСЬКОГО УНІВЕРСИТЕТУ

№ 2 (65), 2021
HERALD

OF THE ODESSA NATIONAL

MARITIME UNIVERSITY № 2 (65), 2021

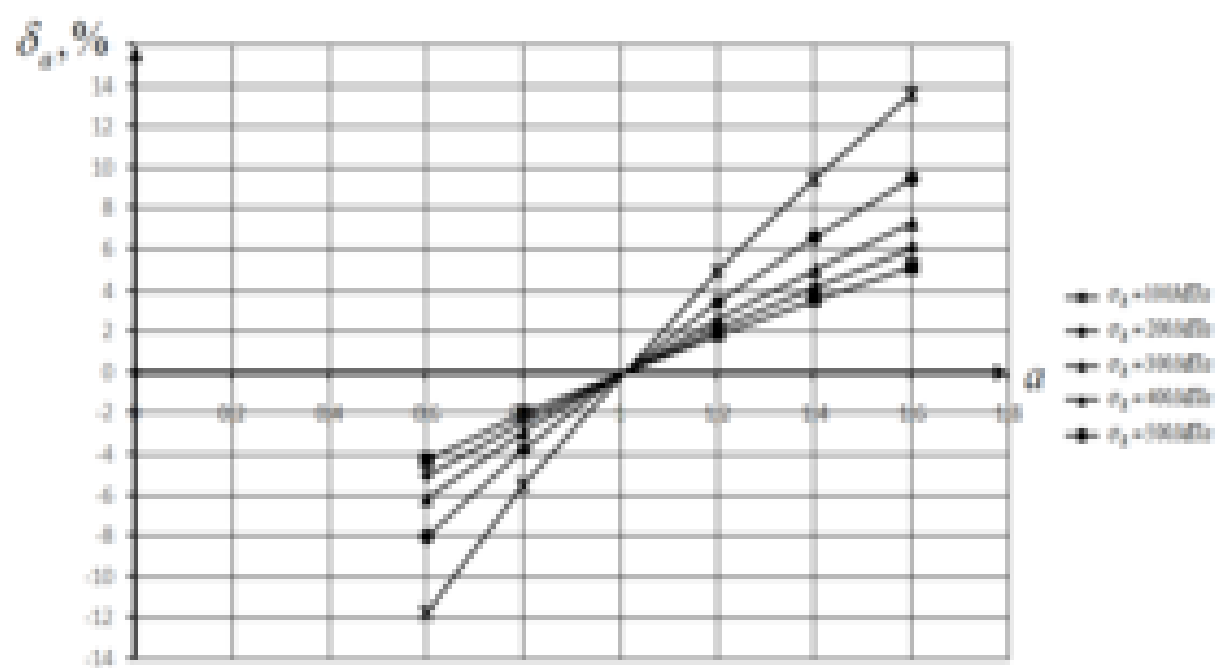

Рис. 2. Зависимости отклонения разрушающего напряжения $\delta_{a}$ от суммы накопленных повреждений а при скорости роста напряжений $\alpha=500$ Па/цикл

\section{Выводы:}

1. Приведенные на рис. 1 и 2 графики свидетельствуют о том, что с увеличением скорости $\alpha$ отклонения разрушающих напряжений $\delta_{a}$ независимо от предела выносливости возрастают.

2. Их наибольшие значения соответствуют максимальным скоростям увеличения нагрузки и в самом неблагоприятном случае не превышают 14 \% (при значении предела выносливости 100 МПа).

\section{ЛІТЕРАТУРА}

1. Коллинз, Дж. Повреждение материалов в конструкииях. Анализ. Предсказание. Предотвращение. - М.: Мир, 1984. $624 c$.

2. Коноплёв, А.В., Селюкова, Е.В. Определение предела выносливости деталей машин методом Про с учётом взаимосвязи между его параметрами // Детали машин: Респ. межвед. науч.-техн. сб. - 1988. - Bbin. 40. - C. 102-104. 


\section{REFERENCES}

1. Kollinz, Dg. Povreghdenie materialov v konstrukciyah. Analiz. Predskazanie. Predotvrashenie. [Damage to materials in structures. Analysis. Prediction. Prevention.] - M.: Mir, 1984. - 624l.

2. Konoplev, A.V., Selukova, E.V. Opredelenie predela vinoslivosti detalei mashin metodom Pro s uchetom vzaimosvyazi megdu ego parametrami [Determination of the endurance limit of machine parts using the Pro method, taking into account the relationship between its parameters]. Detali mashin: Respondenciya meghvedomstvennogo nauchno-tehnicheskogo sbornika (Machine details: Response to an interdepartmental scientific and technical digest). 1987. - Rel. 40. - 102-104 l. [in Russian].

Стаття надійшла 15.03.2021

Посилання на статтю: Коноплёв А.В., Шумило А.Н., Чередарчук Н.И., Галевский В.В., Кононова О.Н., Рожко Е.К., Арпентьева В.А. Оценка влияния скорости увеличения нагрузки на точность определения разрушающего напряжения с помощью гипотезы линейного суммирования усталостных повреждений // Вісник Одеського національного морського університету: 3б. наук. праць, 2021. № 2(65). C. 120-127. DOI 10.47049/ 2226-1893-20212-120-127.

Article received 15.03.2021

Reference a JournalArtic: Konoplev A.V., Shumilo A.N., Cheredarchuk N.I., Halevskyi V.V., Kononova O.N., Rozhko E.K., Arpentieva V.O. Evaluation of the effect of the rate of increase in the load on the accuracy of deter-mining the breaking tensions using the hypothesis of linear summation of fatigue damages // Herald of the Odessa national maritime university, 2021. № 2(65). C. 120-127. DOI 10.47049/ 2226-1893-2021-2-120-127. 